\section{Two-dimensional Fourier Transform Technique for analysing Random Structures}

Is the course of a number of analyses of random interstratification of layers, it has been shown that a one-dimensional Fourier transform can be calculated which gives a clue to the correct sequence of layers' ${ }^{1,2}$.

This method can readily be generalized to two and three dimensions. Suppose we have scattering material, for example, in the form of layers (this is not an essential feature of the method), and that this can be stacked in two or more ways. Suppose that these manners of stacking are mixed together randomly. The result will be an X-ray diagram which consists partly of sharp spots, and partly of diffuse bands. Viewed in three dimensions, the layers will give rise to a series of regularly packed reciprocal rods. These will have a distribution of intensity along them depending on the structure of the layer, and this distribution will further be modified by the nature of the superposition of layers.

Let us consider any plane in reciprocal space, say the (h0l) plane, where (001) is the basal plane. The intensity function of such a partially random crystal as we are considering will have a strict periodicity in the $h$ direction, but will be non-periodic in the $l$ direction. It is now possible to generalize the onedimensional Fourier transform which one of us has described ${ }^{2}$ into a two-dimensional transform, consisting of an infinite band, periodic in the $a$ direction, and non-repeating in the $c$ direction. This function will have maxima at points corresponding to unitcell repeat distances which are present in the random crystal. These unit-cell repeat distances will be shown vectorially. Furthermore, 'combination peaks' will show the degree of randomness in the mixture, as has been described for the one-dimensional case ${ }^{2}$.

The result of this will be to give a vector diagram showing the nature of the various combinations of translations which occur. Fig. 1 shows such a diagram for random mixtures of $(a)$ a $15 \AA$. spacing of $\frac{1}{6} a$ translation, and a $17 \frac{1}{2} \AA$. spacing of $\frac{1}{6} a$ trans-

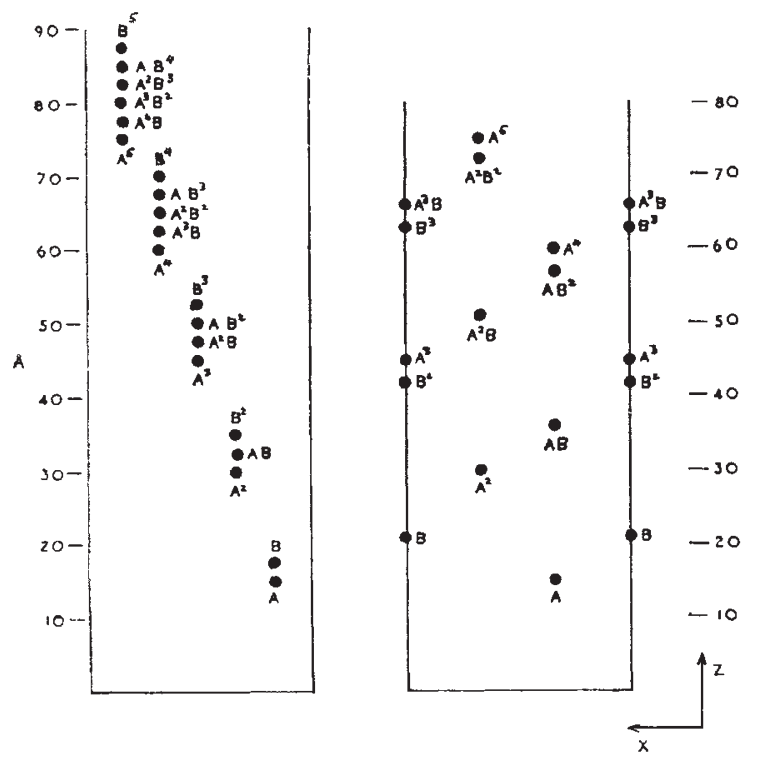

Fig. 1. Theoretical periodograms for two mixtures

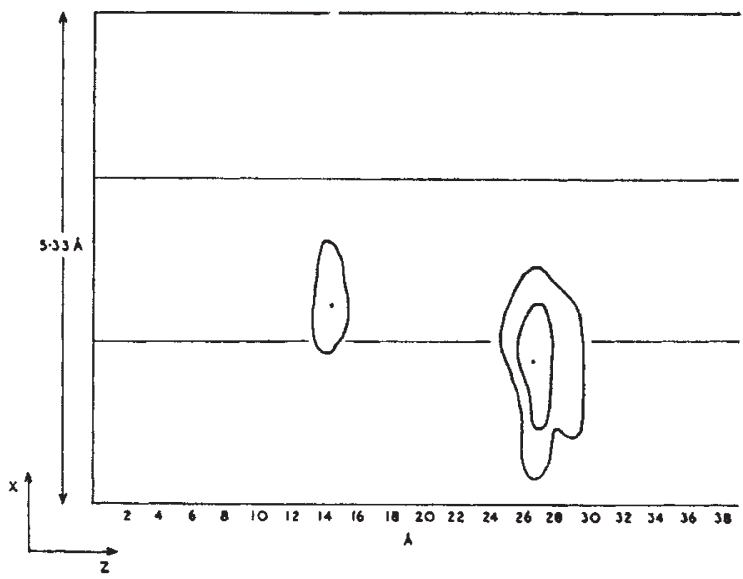

Fig. 2. A periodogram for a $\mathrm{C}_{6} \mathrm{H}_{13} \mathrm{NH}_{\mathbf{2}}$-vermiculite complex

lation; (b) a $15 \AA$. spacing of $\frac{1}{3} a$ translation and a $21 \AA$. spacing of 0 translation.

Fig. 2 shows an actual example of a Fourier transform calculated by this method. It is for a vermiculite complexed with $\mathrm{C}_{6} \mathrm{H}_{13} \mathrm{NH}_{2}$. It shows peaks at $14 \AA$. and $26.5 \AA$., both corresponding to translation of about $\frac{1}{3} a$.

In contrast to an ordinary Fourier synthesis, a diagram of this sort gives the periodicities associated with the crystal, and it may therefore conveniently be called a periodogram.

Douglas M. C. MacEwan

H. H. SUThERLAND

Carnegie Laboratory of Physics, Queen's College, Dundeo.

1 MacEwan, Douglas M. C., Nature, 171, 616 (1953).

MacEwan, Douglas M. C., Koll.-Z., 149, 96 (1956).

\section{METALLURGY}

\section{Creep of Metals under Simple Shear}

IN a paper published last year', we showed with polycrystalline lead, subjected to a constant simple shear stress, that the $t^{1 / 3}$ law of creep held very closely within a certain range of strain, the average error being about 0.25 per cent of the range in question. We recorded, however, that for low strains, below about 0.03 with a particular lead, the law was not obeyed, the strain varying more rapidly than was indicated by the law. At high strains, too, the law fails owing to recrystallization, but to comment on that is not the object of the present communication.

Using a more sensitive recording device, we have since found that, for the lower strains in question, the strain under simple shear stress varies precisely as $t^{1 / 2}$. Thus with a lead for which the $t^{1 / 3}$ regime begins at a strain of 0.035 in the range of strain from 0.012 to 0.035 , the $t^{1 / 2}$ law, fitted by least squares, holds with an average error of 0.25 per cent of the range, which is about the error of observation.

This $t^{1 / 2}$ law has been confirmed with leads differing in the small amounts of foreign metal contained, and with cadmium. For the creep under reversed stress, which can be accurately measured by the 